\title{
Mechanisms of action of Methylthioadenosine: pathways implicated in neuroprotection in models of Multiple Sclerosis and other neurological diseases
}

\author{
B Moreno ${ }^{1 *}$, A Di Penta ${ }^{1}$, M Kerschensteiner ${ }^{2}$, C Matute $^{3}$, V Ceña ${ }^{4}$, I Lopez ${ }^{5}$, J Alberch ${ }^{6}$, J Parent ${ }^{7}$, J Serra ${ }^{8}$, \\ O Errea ${ }^{1}$, P Villoslada ${ }^{1}$
}

From 5th European Workshop on Immune-Mediated Inflammatory Diseases

Sitges-Barcelona, Spain. 1-3 December 2010

\section{Background}

Methylthioadenosine (MTA) has anti-oxidant and antiproliferative properties and was shown to induce cell protection in hepatic cells. We previously demonstrated that exert immunomodulatory and neuroprotective effects in the animal model of Multiple Sclerosis (MS) and other neurological diseases like Parkinson disease, stroke and Epilepsy.

\section{Objective}

To study the mechanisms of action and different pathways implicated in the neuroprotective effect of MTA in neurological diseases.

\section{Methods}

RN22 (Schwnoma cell line) and PC12 (Pheochromocytoma cell line) were used to test the neuroprotective activity of MTA against stress in RN22 and to differentiate neurites in PC12. BV2 cells were used to test the effect of MTA in microglia. Organotypic cerebellum cultures were used to determine MTA effect in demyelination/remyelination. Luminex technology, western blot and ELISA were used in order to study the phosphorylated state of different pathways (AkT/PKB, ERK/MAPK, P38/SAPK or STAT3) and to determine the amount of different cytokines (IL- $1 \beta$ and TNF- $\alpha$ ). Ros determination was also done by fluorescence determination.

\section{Results}

In vitro studies revealed that MTA protection against different stresses and its capacity to differentiate neurites implies pathways like ERK/MAPK, P38/SAPK or STAT3. MTA neuroprotective capacity is also related with its ability to reduce ROS production and oxidative stress. MTA was shown to protect against demyelination in cerebellum organotypic cultures treated with LPS or Lysolecithin.

\section{Conclusions}

MTA is neuroprotective in models of MS, Parkinson disease, stroke or Epilepsy. This neuroprotective effect depends on its capacity to protect against demyelination, its anti-oxidant effect and the activation of pathways related with protection against stress and production of neurite differentiation.

\section{Author details}

${ }^{1}$ Dept. of Neuroscience, Institut of Biomedical research August Pi Sunyer (IDIBAPS), Hospital Clinic of Barcelona, Spain. ${ }^{2}$ University of Munich, Germany. ${ }^{3}$ University of Basque Country, Spain. ${ }^{4}$ University of Castilla la Mancha, Spain.

${ }^{5} \mathrm{CIMA}$ - University of Navarra, Spain. ${ }^{6}$ University of Barcelona, Spain.

${ }^{7}$ University of Michigan, USA. ${ }^{8}$ Neuroscience Technologies, Spain.

Published: 25 November 2010

doi:10.1186/1479-5876-8-S1-P28

Cite this article as: Moreno et al:: Mechanisms of action of

Methylthioadenosine: pathways implicated in neuroprotection in models of Multiple Sclerosis and other neurological diseases. Journal of Translational

Medicine 2010 8(Suppl 1):P28. 